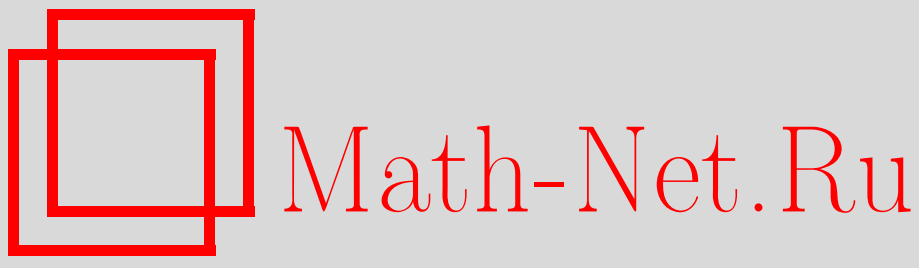

В. Е. Зотеев, Д. Н. Попова, Определение динамических характеристик нелинейных диссипативных систем на основе стохастического разностного уравнения, Вестн. Сам. гос. техн. ун-та. Сер. Физ.-мат. науки, 2006, выпуск 42, 162-168

DOI: https://doi.org/10.14498/vsgtu427

Использование Общероссийского математического портала Math-Net.Ru подразумевает, что вы прочитали и согласны с пользовательским соглашением

http://www . mathnet.ru/rus/agreement

Параметры загрузки:

IP: 18.234 .156 .22

26 апреля 2023 г., 14:07:41

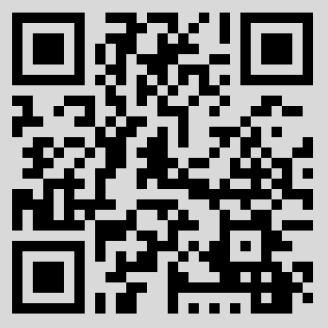




\title{
ОПРЕДЕЛЕНИЕ ДИНАМИЧЕСКИХ ХАРАКТЕРИСТИК НЕЛИНЕЙНЫХ ДИССИПАТИВНЫХ СИСТЕМ НА ОСНОВЕ СТОХАСТИЧЕСКОГО РАЗНОСТНОГО УРАВНЕНИЯ
}

\begin{abstract}
Рассматривается линейно параметрическая дискретная модель свободных колебаний механической системы с нелинейными диссипативными силами в форме стохастического разностного уравнения; предлагается эффективный численный итерационный метод среднеквадратичного оиенивания коэффициентов стохастического разностного уравнения; приводятся результать численноаналитических исследований помехозащищенности алгоритма вычисления динамических характеристик диссипативной системы на основе среднеквадратичных оценок коэффициентов разностного уравнения.
\end{abstract}

Техническое состояние механической системы неразрывно связано с характеристиками рассеяния энергии колебаний. При этом одним из информативных признаков технического состояния является степень нелинейности действующих в системе диссипативных сил.

Современный уровень развития средств вычислений позволяет эффективно использовать статистические методы обработки экспериментальных данных при параметрической идентификации системы. Однако традиционные методы определения характеристик рассеяния энергии при колебаниях в большинстве своем не позволяют в полной мере использовать преимущества новых информационных технологий и в этом смысле они “морально” устарели. Решить проблему эффективного внедрения современных методов и средств вычислений в практику экспериментальных исследований демпфирующих свойств механических систем можно только на основе новых математических моделей. Этим требованиям в полной мере удовлетворяют линейно параметрические дискретные модели (ЛПДМ), рекуррентно описывающие временные последовательности мгновенных значений свободных колебаний диссипативной системы [1]. Применение таких моделей позволяет определять динамические характеристики через среднеквадратичные оценки коэффициентов ЛПДМ, вычисленные на основе статистической обработки выборки экспериментальных данных. Такой способ принципиально отличается от традиционных методов нахождения динамических характеристик диссипативной системы [2], он позволяет обеспечить высокую точность оценивания за счет эффективного использования современных средств вычислений и их математического обеспечения.

В данной работе рассматривается метод определения характеристик рассеяния энергии при колебаниях нелинейной диссипативной системы, в основе которого лежит итерационная процедура среднеквадратичного оценивания коэффициентов ЛПДМ в форме стохастического разностного уравнения. Разработка этого метода включает решение следующих основных задач: построение линейно параметрической дискретной модели свободных колебаний систем с диссипативными силами, пропорциональными $n$ - степени скорости движения, в форме стохастического разностного уравнения; описание алгоритма определения динамических характеристик на основе среднеквадратичных оценок коэффициентов стохастического разностного уравнения; разработка и исследование эффективности итерационного метода среднеквадратичного оценивания коэффициентов ЛПДМ.

Рассмотрим построение ЛПДМ в форме рекуррентной формулы, связывающей три последовательных мгновенных значения непрерывной функции, описывающей свободные колебания в диссипативной системе с диссипативными силами, пропорциональными $n$ - степени скорости движения: $\tilde{y}(t)=\frac{a_{0}}{1+\frac{\delta_{0}}{T} t+\left(1-\frac{n}{2}\right)\left(\frac{\delta_{0}}{T} t\right)^{2}} \cos \left(\omega t+\psi_{0}\right)$, где $\omega$ и $\delta_{0}-$ частота и декремент колебаний; $n$ - характеристика нелинейности; $T=2 \pi \omega^{-1}-$ период колебаний; $a_{0}$ и $\psi_{0}$ - начальные амплитуда и фаза колебаний. Полагая $t=\tau k, k=0,1,2, \ldots$, где $\tau-$ период равномерной дискретиза-

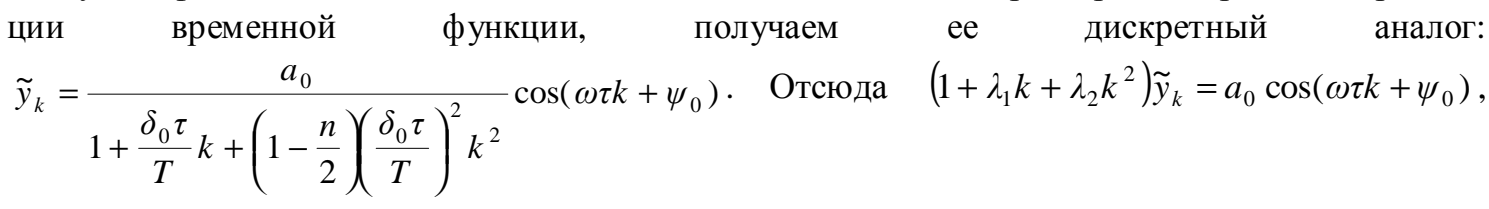


где $\lambda_{1}=\frac{\delta_{0} \tau}{T}$ и $\lambda_{2}=\left(1-\frac{n}{2}\right) \lambda_{1}{ }^{2}$. Применяя к обеим частям данного выражения $z$-преобразование, получаем $Z\left\{\left(1+\lambda_{1} k+\lambda_{2} k^{2}\right) \tilde{y}_{k}\right\}=a_{0} \frac{\cos \psi_{0}-z^{-1} \cos \left(\omega \tau-\psi_{0}\right)}{1-\lambda_{0} z^{-1}+z^{-2}}, \quad$ где $\quad \lambda_{0}=2 \cos \omega \tau . \quad$ Отсюда $Z\left\{\left(1+\lambda_{1} k+\lambda_{2} k^{2}\right) \tilde{y}_{k}\right\}-z^{-1} \lambda_{0} Z\left\{\left(1+\lambda_{1} k+\lambda_{2} k^{2}\right) \tilde{y}_{k}\right\}+z^{-2} Z\left\{\left(1+\lambda_{1} k+\lambda_{2} k^{2}\right) \tilde{y}_{k}\right\}=a_{0} \cos \psi_{0}-z^{-1} a_{0} \cos \left(\omega \tau-\psi_{0}\right)$. Возвращаясь в пространство оригиналов, используя первую теорему смещения: $z^{-r} Z\left\{\tilde{y}_{k}\right\}=\tilde{y}_{k-r}$, $r=0,1,2$, при условии, что $\tilde{y}_{k-r}=0$ при $k-r<0$, получаем разностное уравнение вида

$$
\begin{aligned}
\left(1+\lambda_{1} k+\lambda_{2} k^{2}\right) \tilde{y}_{k} & -\lambda_{0}\left[1+(k-1) \lambda_{1}+(k-1)^{2} \lambda_{2}\right] \tilde{y}_{k-1}+\left[1+(k-2) \lambda_{1}+(k-2)^{2} \lambda_{2}\right] \tilde{y}_{k-2}= \\
& =\delta_{k} a_{0} \cos \psi_{0}-\delta_{k-1} a_{0} \cos \left(\omega \tau-\psi_{0}\right), k=0,1,2, \mathrm{~K},
\end{aligned}
$$

где $\delta_{k}=\left\{\begin{array}{l}1, \text { при } k=0 ; \\ 0, \text { при } k \neq 0\end{array} \quad\right.$ - символ Кронекера.

При $k=0$ и $k=1$ соответственно получаем:

$$
\tilde{y}_{0}=a_{0} \cos \psi_{0}, \quad \tilde{y}_{1}=\frac{a_{0}}{1+\lambda_{1}+\lambda_{2}}\left(\cos \omega \tau \cos \psi_{0}-\sin \omega \tau \sin \psi_{0}\right) .
$$

При $k \geq 2$ приходим к разностному уравнению второго порядка: $\left(1+\lambda_{1} k+\lambda_{2} k^{2}\right) \tilde{y}_{k}-$ $-\lambda_{0}\left[1+(k-1) \lambda_{1}+(k-1)^{2} \lambda_{2}\right] \tilde{y}_{k-1}+\left[1+(k-2) \lambda_{1}+(k-2)^{2} \lambda_{2}\right] \tilde{y}_{k-2}=0, \quad$ из которого после простых преобразований получаем линейно параметрическую дискретную модель, рекуррентно описывающую временную последовательность дискретных значений исследуемой функции:

$$
\lambda_{0} \tilde{y}_{k-1}-\lambda_{1}\left[k \tilde{y}_{k}+(k-2) \tilde{y}_{k-2}\right]-\lambda_{2}\left[k^{2} \tilde{y}_{k}+(k-2)^{2} \tilde{y}_{k-2}\right]+\lambda_{3}(k-1) \tilde{y}_{k-1}+\lambda_{4}(k-1)^{2} \tilde{y}_{k-1}=\tilde{y}_{k}+\tilde{y}_{k-2} \text {. }
$$

Коэффициенты в модели (2) связаны с динамическими характеристиками соотношениями:

$$
\lambda_{0}=2 \cos \omega \tau, \quad \lambda_{1}=\frac{\delta_{0} \tau}{T}, \quad \lambda_{2}=\left(1-\frac{n}{2}\right) \lambda_{1}^{2}, \quad \lambda_{3}=\lambda_{0} \lambda_{1}, \quad \lambda_{4}=\lambda_{0} \lambda_{2} .
$$

При обработке экспериментальной виброграммы свободных колебаний формируется выборка результатов наблюдений $y_{k}, k=\overline{0, N-1}$, где $N$ - объем выборки. Результаты наблюдений $y_{k}$ содержат аддитивную случайную помеху $\varepsilon_{k}: y_{k}=\tilde{y}_{k}+\varepsilon_{k}$, где $\tilde{y}_{k}-$ дискретные значения, соответствующие теоретической модели колебаний, и, следовательно, соотношениям (1) и (2). Обозначая

$$
\lambda_{5}=a_{0} \cos \psi_{0}, \quad \lambda_{6}=-a_{0} \sin \omega \tau \sin \psi_{0},
$$

с учетом случайной помехи в отсчетах ординат колебаний, линейно параметрическую дискретную модель в форме стохастического разностного уравнения можно представить в виде

$$
\left\{\begin{array}{l}
y_{0}=\lambda_{5}+\varepsilon_{0}, \\
y_{1}=\lambda_{0} \frac{y_{0}}{2}-\lambda_{1} y_{1}-\lambda_{2} y_{1}+\lambda_{6}-\frac{\lambda_{0}}{2} \varepsilon_{0}+\left(1+\lambda_{1}+\lambda_{2}\right) \varepsilon_{1}, \\
y_{k}+y_{k-2}=\lambda_{0} y_{k-1}-\lambda_{1}\left[k y_{k}+(k-2) y_{k-2}\right]-\lambda_{2}\left[k^{2} y_{k}+(k-2)^{2} y_{k-2}\right]+\lambda_{3}(k-1) y_{k-1}+ \\
\quad+\lambda_{4}(k-1)^{2} y_{k-1}+\eta_{k}, k=\overline{2, N-1}, \\
\eta_{k}=\left[1+(k-2) \lambda_{1}+(k-2)^{2} \lambda_{2}\right] \varepsilon_{k-2}-\lambda_{0}\left[1+(k-1) \lambda_{1}+(k-1)^{2} \lambda_{2}\right] \varepsilon_{k-1}+ \\
+\left[1+k \lambda_{1}+k^{2} \lambda_{2}\right] \varepsilon_{k}, k=\overline{2, N-1 .}
\end{array}\right.
$$

В матричной форме линейно параметрическая дискретная модель, описывающая свободные колебания систем с диссипативными силами общего вида при случайной аддитивной помехе в отсчетах наблюдений, имеет вид 


$$
\left\{\begin{array}{l}
b=F \lambda+\eta \\
\eta=P \varepsilon
\end{array}\right.
$$

или в развернутой форме обобщенной регрессионной модели

$$
\begin{aligned}
& b_{i}=\lambda_{0} f_{i 1}+\lambda_{1} f_{i 2}+\lambda_{2} f_{i 3}+\lambda_{3} f_{i 4}+\lambda_{4} f_{i 5}+\lambda_{5} f_{i 6}+\lambda_{6} f_{i 7}+\eta_{i}, \\
& \eta_{i}=\varepsilon_{0} p_{i 1}+\varepsilon_{1} p_{i 2}+\varepsilon_{2} p_{i 3}+\mathrm{K}+\varepsilon_{N-2} p_{i, N-1}+\varepsilon_{N-1} p_{i N},
\end{aligned}
$$

Здесь $\lambda=\left(\lambda_{0}, \lambda_{1}, \lambda_{2}, \lambda_{3}, \lambda_{4}, \lambda_{5}, \lambda_{6}\right)^{T} \quad-\quad$ вектор неизвестных коэффициентов ЛПДМ; $\varepsilon=\left(\varepsilon_{0}, \varepsilon_{1}, \mathrm{~K}, \varepsilon_{N-1}\right)^{T}-N$-мерный вектор случайной помехи в результатах наблюдений; $\eta=\left(\eta_{1}, \eta_{2}, \mathrm{~K}, \eta_{N}\right)^{T}-N$-мерный вектор эквивалентного возмущения в стохастическом разностном уравнении; $b=\left(y_{0}, y_{1}, y_{0}+y_{2}, \mathrm{~K}, y_{N-3}+y_{N-1}\right)^{T}-N$-мерный вектор правой части; $F$ - матрица регрессоров размера $N \times 7$, столбцы которой описываются формулами:

$f_{i 1}=\left(0, \frac{y_{0}}{2}, y_{1}, y_{2}, \mathrm{~K}, y_{k-2}, \mathrm{~K}, y_{N-2}\right)^{T}$ $k=\overline{3, N}$,

$f_{i 2}=\left(0,-y_{1},-2 y_{2},-\left(y_{1}+3 y_{3}\right), \mathrm{K},-\left[(k-3) y_{k-3}+(k-1) y_{k-1}\right], \mathrm{K},-\left[(N-3) y_{N-3}+(N-1) y_{N-1}\right]\right)^{T}, \quad k=\overline{3, N}$, $f_{i 3}=\left(0,-y_{1},-4 y_{2},-\left(y_{1}+9 y_{3}\right), \mathrm{K},-\left[(k-3)^{2} y_{k-3}+(k-1)^{2} y_{k-1}\right] \mathrm{K},-\left[(N-3)^{2} y_{N-3}+(N-1)^{2} y_{N-1}\right]\right)^{T} ; k=\overline{3, N}$, $f_{i 4}=\left(0,0, y_{1}, 2 y_{2}, 3 y_{3}, \mathbf{K},(k-2) y_{k-2}, \mathbf{K},(N-2) y_{N-2}\right)^{T}$, $f_{i 5}=\left(0,0, y_{1}, 2 y_{2}, 9 y_{3}, \mathrm{~K},(k-2)^{2} y_{k-2}, \mathrm{~K},(N-2)^{2} y_{N-2}\right)^{T}$, $k=\overline{3, N}$, $f_{i 6}=(1,0, \mathrm{~K}, 0)^{T}, f_{i 7}=(0,1,0, \mathrm{~K}, 0)^{T}$;

$P$ - матрица размера $N \times N$ в стохастическом уравнении эквивалентного возмущения - нижняя треугольная, ленточная, трехдиагональная. Первый столбец матрицы $P$ имеет вид: $p_{i 1}=\left(1,-\frac{\lambda_{0}}{2}, 1,0, \mathrm{~K}, 0\right)^{T}, i=\overline{1, N}$. Остальные столбцы матрицы $P, j=\overline{2, N}$, описываются формулами:

$$
p_{i j}= \begin{cases}0, & i<j u i \geq j+3, \\ 1+(j-1) \lambda_{1}+(j-1)^{2} \lambda_{2}, & i=j u \quad i=j+2, \\ -\lambda_{0}\left[1+(j-1) \lambda_{1}+(j-1)^{2} \lambda_{2}\right], & i=j+1 .\end{cases}
$$

Рассмотрим процедуру вычисления характеристик рассеяния энергии при колебаниях систем с силами трения, пропорциональными $n$ - степени скорости движения, в основе которой лежат среднеквадратичные оценки коэффициентов разностного уравнения (5). Исходными данными являются результаты измерений $y_{k}, k=\overline{0, N-1}$, мгновенных значений виброграммы свободных колебаний. $N$ - объем выборки результатов измерений с периодом дискретизации $\tau$. Будем предполагать, что случайные возмущения $\varepsilon_{k}, k=\overline{0, N-1}$, в результатах измерений $y_{k}$ имеют нулевое математическое ожидание $M\left[\varepsilon_{k}\right]=0$, дисперсию $\sigma_{\varepsilon}^{2}$ и не коррелированны между собой: матрица дисперсий-ковариаций $V[\varepsilon]=\sigma_{\varepsilon}^{2} E$, где $E$ - единичная матрица.

На первом этапе по приведенным выше формулам, описывающим элементы стохастического разностного уравнения (7), вычисляются элементы матрицы $F=\left(f_{i j}\right), j=\overline{1,7}$, и правой части $b=\left(b_{i}\right), i=\overline{1, N}$, переопределенной системы линейных алгебраических уравнений относительно коэффициентов $\lambda_{j}, j=\overline{1,7}$. На втором этапе вычисляются среднеквадратичные оценки коэффициентов стохастического разностного уравнения. Данный этап является определяющим в процессе формирования погрешности оценок динамических характеристик. От того, на сколько обоснованно и корректно будет выбран метод среднеквадратичного оценивания, существенно зависит точность вычисления коэффициентов ЛПДМ. На заключительном этапе с учетом известных соотношений (3) по среднеквадратичным оценкам $\hat{\lambda}_{i}$ коэффициентов ЛПДМ вычис- 
ляются динамические характеристики диссипативной системы, в том числе показатель нелинейности силы трения:

$$
\omega=\frac{1}{\tau} \arccos \frac{\hat{\lambda}_{0}}{2}, \quad \delta_{0}=\frac{2 \pi \hat{\lambda}_{1}}{\omega \tau}=\frac{2 \pi \hat{\lambda}_{1}}{\arccos \left(0,5 \hat{\lambda}_{0}\right)}, \quad n=2\left(1-\frac{\hat{\lambda}_{2}}{\hat{\lambda}_{1}^{2}}\right) .
$$

Используемая линейно параметрическая дискретная модель с учетом формул (4) позволяет также оценивать начальную амплитуду и фазу колебаний:

$$
\mu=-\frac{2 \hat{\lambda}_{6}}{\sqrt{4-\hat{\lambda}_{0}^{2}}}, \quad a_{0}=\sqrt{\hat{\lambda}_{5}^{2}+\mu^{2}}, \quad \psi_{0}=\operatorname{arctg} \frac{\mu}{\hat{\lambda}_{5}} .
$$

Применение статистических методов при вычислении коэффициентов линейно параметрической дискретной модели $\lambda_{j}$ позволяет существенно повысить точность оценивания. Однако вычисление среднеквадратичных оценок $\hat{\lambda}_{j}$ по обобщенной регрессионной модели, которая описывается уравнением (7): $\sum_{i=1}^{N}\left[b_{i}-\sum_{j=0}^{6} \lambda_{j} f_{i, j+1}\right]^{2}=\sum_{i=1}^{N} \eta_{i}^{2} \Rightarrow \min$, приводит к значительному асимптотическому смещению в оценках из-за большой корреляции между отсчетами эквивалентного случайного возмущения $\eta_{i}$ и элементами матрицы $f_{i j}, j=\overline{1,3}$, [4]. Этот существенный недостаток устраняется за счет использования итерационной процедуры при вычислении среднеквадратичных оценок.

При невырожденной матрице $P$ после преобразования обобщенной регрессионной модели (7) получаем $P_{\lambda}^{-1} b=P_{\lambda}^{-1} F \lambda+\varepsilon$. Вычисление среднеквадратичных оценок коэффициентов $\lambda_{j}$ на основе минимизации функционала $J_{\lambda}=\left\|P_{\lambda}^{-1} b-P_{\lambda}^{-1} F \lambda\right\|^{2}=\|\varepsilon\|^{2} \Rightarrow \min$ позволяет существенно уменьшить асимптотическое смещение, связанное с корреляцией между случайным возмущением и результатами наблюдений, и тем самым повысить точность вычислений динамических характеристик. Так как элементы матрицы $P_{\lambda}^{-1}$ зависят от $\lambda_{0}, \lambda_{1}$ и $\lambda_{2}$, то, в общем случае, минимизация функционала $J_{\lambda}$ приводит к решению системы нелинейных относительно $\lambda_{j}$ уравнений. Рассмотрим итерационную процедуру вычисления среднеквадратичных оценок коэффициентов $\lambda_{j}$, реализующую метод последовательных приближений. На первом шаге вычисляются первоначальные МНК - оценки $\lambda_{j}^{(0)}:\|b-F \lambda\|^{2}=\|\eta\|^{2} \Rightarrow \min$. Затем по формулам (9) вычисляется приближение матрицы $P_{\lambda} \approx P_{\lambda}^{(1)}\left(\lambda_{0}^{(0)}, \lambda_{1}^{(0)}, \lambda_{2}^{(0)}\right)$, преобразуется обобщенная регрессионная модель (7) и находятся новые (уточненные) оценки $\lambda_{j}^{(1)}$ коэффициентов ЛПДМ. Эти оценки используются для вычисления второго приближения матрицы $P_{\lambda} \approx P_{\lambda}^{(2)}\left(\lambda_{0}^{(1)}, \lambda_{1}^{(1)}, \lambda_{2}^{(1)}\right)$ и т.д. Процесс уточнения повторяется до тех пор, пока не будет выполнено условие останова $\left\|\lambda^{(i)}-2 \lambda^{(i-1)}+\lambda^{(i-2)}\right\|<0,01\left\|\lambda^{(i-1)}\right\|$. Найденные на последней итерации оценки $\lambda^{(i)}$ принимаются за истинные значения коэффициентов ЛПДМ и по ним далее вычисляются динамические характеристики диссипативной системы.

Проведены численно-аналитические исследования сходимости итерационной процедуры метода определения динамических характеристик на основе среднеквадратичного оценивания коэффициентов стохастического разностного уравнения. Численный эксперимент был организован следующим образом. Генерировалась выборка $\tilde{y}_{k}, k=\overline{0, N-1}$, дискретных значений функции, описывающей свободные колебания в системе с диссипативными силами, пропорциональными $n$ - степени скорости движения, с параметрами $a_{0}=1, \psi_{0}=1, \delta_{0}=0,05, \omega=2 \pi$, $n=1,5$. Период дискретизации $\tau$ был выбран таким образом, что при $T=\frac{2 \pi}{\omega}=1$ отношение $\frac{\tau}{T}=0,2$. Объем выборки составил $N=100$. В отсчеты $\tilde{y}_{k}$, соответствующие точным значениям 
функции, добавлялась аддитивная помеха $\varepsilon_{k}$, значения которой обеспечивали заданную величину мощности помехи $1 \%$ в относительных единицах: $\frac{\|\varepsilon\|}{\|\tilde{y}\|} 100 \%$.

На рис. 1 представлены зависимости относительной погрешности вычисления декремента колебаний (кривая 1) и характеристики нелинейности (кривая 2) от числа итераций. Точки на графиках при $s=0$ соответствуют результатам среднеквадратичного оценивания коэффициентов $\lambda_{j}$ без предварительного преобразования обобщенной регрессионной модели (7).

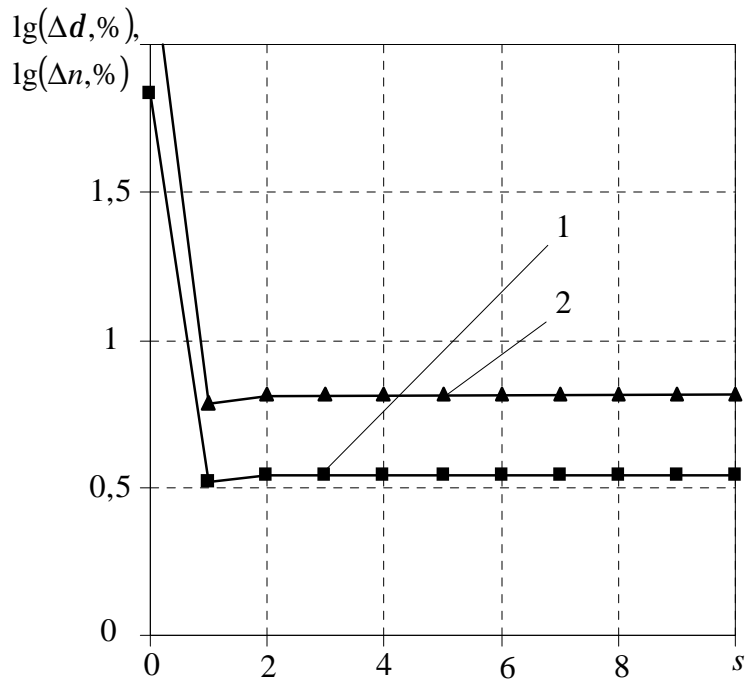

Р и с. 1 Зависимости относительной погрешности вычисления декремента колебаний (1) и характеристики нелинейности (2) от числа итераций

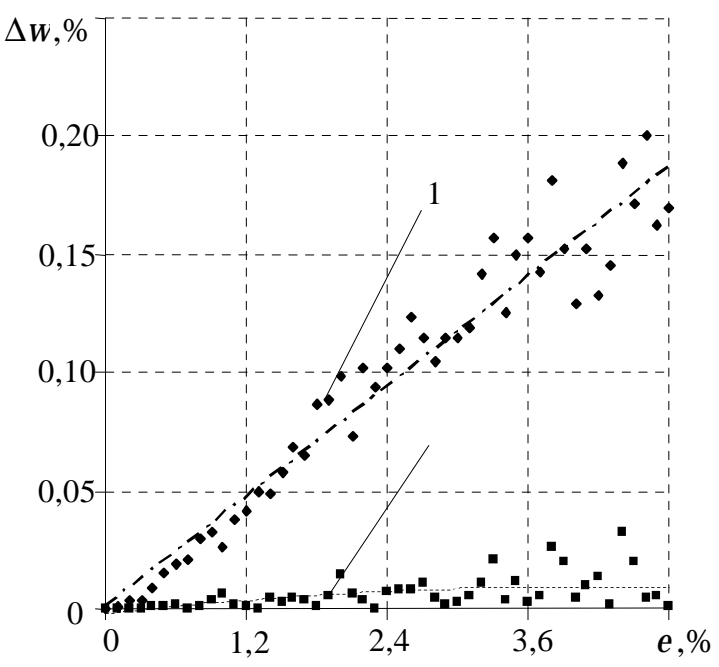

Р и с. 2 Зависимости относительной погрешности вычисления частоты колебаний от величины случайной помехи в результатах наблюдений

По результатам исследований можно сделать следующие выводы. Во-первых, применение итерационного метода вычисления среднеквадратичных оценок позволяет существенно, в десятки раз, уменьшить погрешность вычисления основных диссипативных характеристик - декремента колебаний и характеристики нелинейности. Во-вторых, результаты вычислений практически не изменяются после второй итерации, что свидетельствует о хорошей сходимости метода.

Проведены исследования эффективности предложенного итерационного метода вычисления среднеквадратичных оценок. Цель исследований - сравнить погрешности вычисления динамических характеристик методом наименьших квадратов без преобразования обобщенной регрессионной модели (7) и с применением итерационного численного метода. Для этого, как и в предыдущем эксперименте, моделировалась выборка объема $N=100$ тестового сигнала с параметрами $a_{0}=1, \psi_{0}=1, \delta_{0}=0,05, \omega=2 \pi, \tau=0,2, n=1,5$. В отсчеты тестового сигнала добавлялась случайная помеха, мощность которой изменялась в диапазоне от 0 до 5\%. Для каждой точки эксперимента результаты вычисления относительной погрешности усреднялись по $M=100$ реализациям.

На рис. 2 - 4 представлены зависимости относительной погрешности вычисления частоты, декремента колебаний и характеристики нелинейности от мощности случайной помехи. Точки 1 соответствуют методу наименьших квадратов, примененному к обобщенной регрессионной модели (7) без ее преобразования; точки 2 - итерационному методу вычислений среднеквадратичных оценок коэффициентов ЛПДМ.

Из результатов исследований видно, что погрешность оценивания частоты колебаний достаточно мала даже при использовании метода наименьших квадратов, примененного к обобщенной регрессионной модели (7) без ее преобразования. Зато точность вычисления ДХ при использовании итерационного метода среднеквадратичного оценивания существенно, в десятки, а для декремента колебаний и характеристики нелинейности - в сотни раз выше по сравнению с методом наименьших квадратов, примененному к обобщенной регрессионной модели. 
Таким образом, применение итерационного метода среднеквадратического оценивания коэффициентов стохастического разностного уравнения позволяет существенно увеличить точность вычисления ДХ по сравнению с методом наименьших квадратов для обобщенной регрессионной модели (7) без ее преобразования.

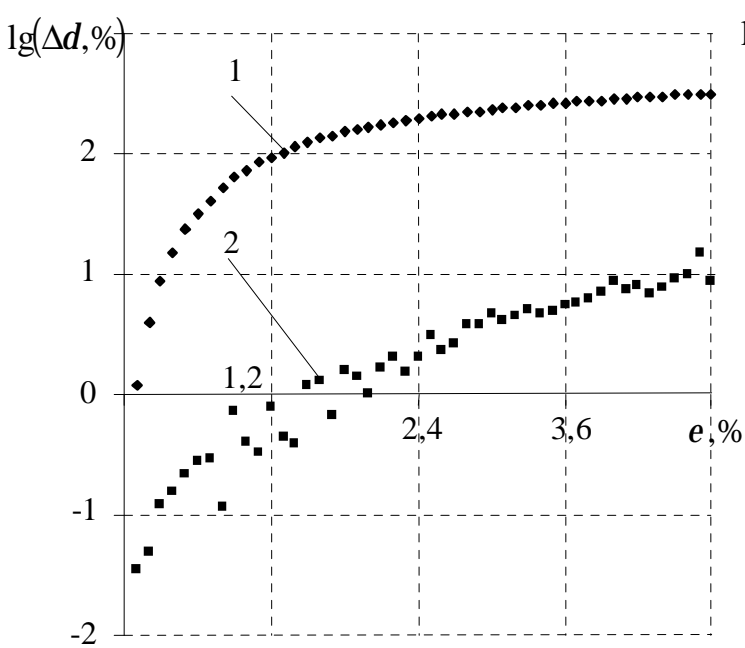

Р и с. 3 Зависимости относительной погрешности вычисления декремента колебаний от величины случайной помехи в результатах наблюдений

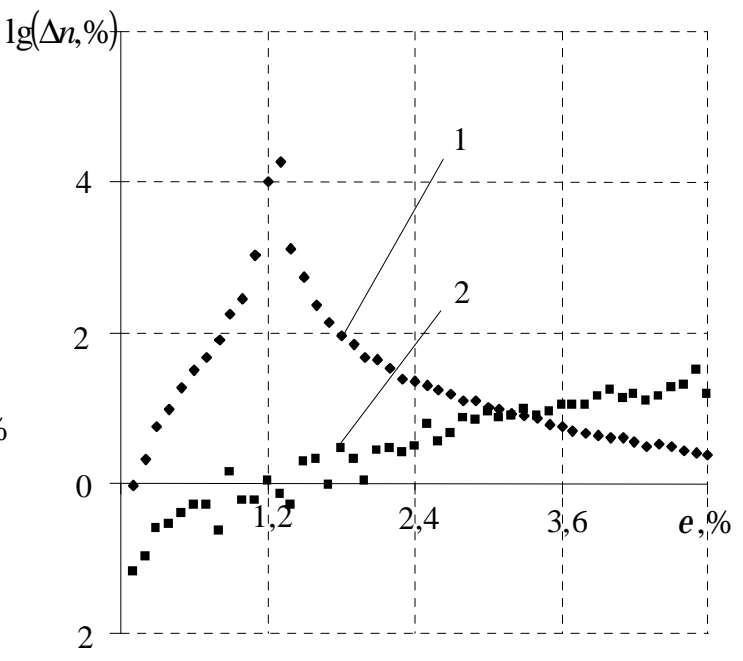

Р и с. 4 Зависимости относительной погрешности вычисления характеристики нелинейности от величины случайной помехи в результатах наблюдений

Проведены численно-аналитические исследования зависимости погрешности вычисления динамических характеристик от периода дискретизации $\tau$ и $N$ при использовании итерационного метода среднеквадратичного оценивания. Моделировались свободные колебания системы с параметрами тестового сигнала: $a_{0}=1, \psi_{0}=1, \delta_{0}=0,05, \omega=2 \pi, n=1,5, \varepsilon=1 \%$. Число усреднений для каждой точки эксперимента: $M=1000$.

На рис. 5 и рис. 6 представлены зависимости относительной погрешности вычисления декремента колебаний и характеристики нелинейности от периода дискретизации при $N=100$. Очевидно, что с уменьшением периода дискретизации погрешность вычислений диссипативных характеристик увеличиваются. Это связано с ухудшением устойчивости вычислений при решении системы нормальных уравнений при среднеквадратичном оценивании.

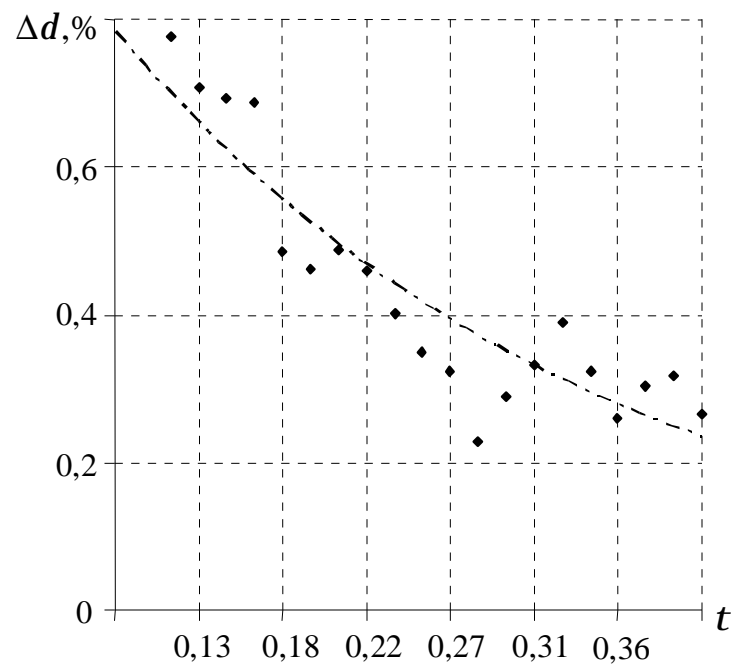

Р и с. 5 Зависимость относительной погрешности вычисления декремента колебаний от периода дискретизации ( $N=100$ )

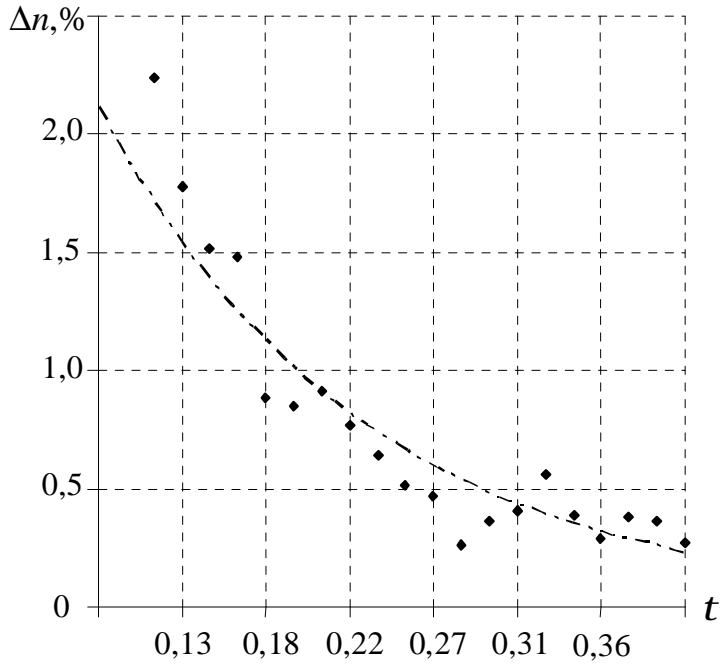

Р и с. 6 Зависимость относительной погрешности вычисления характеристики нелинейности от периода дискретизации ( $N=100$ ) 
На рис. 7 и рис. 8 представлены зависимости относительной погрешности вычисления декремента колебаний и характеристики нелинейности от объема выборки при $\tau=0,2$. Видно, что с увеличением объема выборки результатов измерений ординат свободных колебаний погрешность вычислений уменьшается, причем существенно.

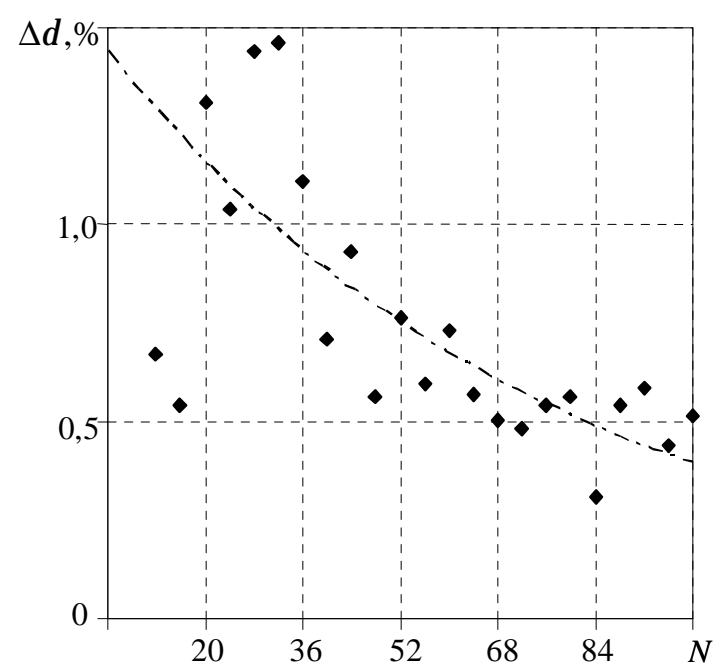

Р и с. 7 Зависимости относительной погрешности вычисления декремента колебаний от объема выборки ( $\tau=0,2)$

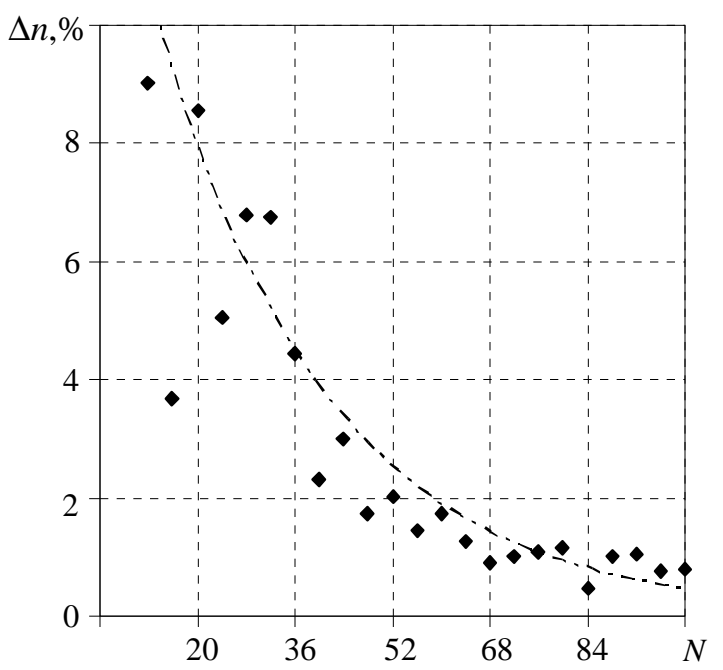

Р и с. 8 Зависимости относительной погрешности вычисления характеристики нелинейности от объема выборки ( $\tau=0,2$ )

Таким образом, разработан эффективный метод определения диссипативных характеристик механических систем с силами трения, пропорциональными $n$ - скорости движения, позволяющий получать оценки декремента колебаний и характеристики нелинейности диссипативной силы с высокой точностью. Этого удалось достигнуть за счет применения линейно параметрических дискретных моделей в форме стохастических разностных уравнений и итерационного метода среднеквадратичного оценивания коэффициентов этого разностного уравнения. Рассмотренный в данной работе метод может быть рекомендован для определения демпфирующих характеристик механических систем с гистерезисным трением.

\section{БИБЛИОГРАФИЧЕСКИЙ СПИСОК}

1. Зотеев B.E. Разработка и исследование линейных дискретных моделей колебаний диссипативных систем // Вестник Самарск. госуд. техн. ун.-та. Серия: Физ.-математ. науки, 1999. № 7. С. 170-177.

2. Писаренко Г.С., Матвеев В.А., Яковлев А.П. Методы определения характеристик колебаний упругих систем. Киев: Наук. думка, 1976. 88 с.

3. Деч Г. Руководство к практическому применению преобразования Лапласа и $Z$-преобразования. М.: Наука, 1971. $288 \mathrm{c}$.

4. Вучков И., Бояджиева Л., Солаков Е. Прикладной линейный регрессионный анализ. М.: Финансы и статистика, 1987. $239 \mathrm{c}$. 